\title{
Generation of radiative knots in a randomly pulsed protostellar jet
}

\section{Dynamics and energetics}

\author{
R. Bonito ${ }^{1,2}$, S. Orlando ${ }^{2}$, G. Peres ${ }^{1,2}$, J. Eislöffel $^{3}$, M. Miceli ${ }^{1,2}$, and F. Favata ${ }^{4}$ \\ 1 Dip. Scienze Fisiche ed Astronomiche, Sez. Astronomia, Università di Palermo, P.zza del Parlamento 1, 90134 Palermo, Italy \\ e-mail: sbonito@astropa.unipa.it \\ 2 INAF - Osservatorio Astronomico di Palermo, P.zza del Parlamento 1, 90134 Palermo, Italy \\ 3 Thüringer Landessternwarte, Sternwarte 5, 07778 Tautenburg, Germany \\ 4 European Space Agency Community Coordination and Planning Office, 8-10 rue Mario Nikis, 75738 Paris Cedex 15, France
}

Received 3 November 2009 / Accepted 9 December 2009

ABSTRACT

\begin{abstract}
Context. Herbig-Haro objects are characterized by a complex knotty morphology detected mainly along the axis of protostellar jets in a wide range of bands: from radio to IR to optical bands, with X-rays knots also detected in the past few years. Evidence of interactions between knots formed in different epochs have been found, suggesting that jets may result from the ejection of plasma blobs from the stellar source.

Aims. We aim at investigating the physical mechanism leading to the irregular knotty structure observed in protostellar jets in different wavelength bands and the complex interactions occurring among blobs of plasma ejected from the stellar source.

Methods. We performed 2D axisymmetric hydrodynamic numerical simulations of a randomly ejected pulsed jet. The jet consists of a train of blobs that ram with supersonic speed into the ambient medium. The initial random velocity of each blob follows an exponential distribution. We explored the ejection rate parameter to derive constraints on the physical properties of protostellar jets by comparing model results with observations. Our model takes the effects of radiative losses and thermal conduction into account. Results. We find that the mutual interactions of blobs ejected at different epochs and with different speeds lead to a variety of plasma components not described by current models of jets. The main features characterizing the randomly pulsed jet scenario are: single high-speed knots, showing a measurable proper motion in nice agreement with optical and X-rays observations; irregular chains of knots aligned along the jet axis and possibly interacting with each other; reverse shocks interacting with outgoing knots; oblique shock patterns produced by the reflection of shocks at the cocoon surrounding the jet. All these structures work together to help determining the morphology of the jet in different wavelength bands. We also find that the thermal conduction plays a crucial role in damping out hydrodynamic instabilities that would develop within the cocoon and that contribute to the jet breaking.
\end{abstract}

Key words. hydrodynamics - Herbig-Haro objects - ISM: jets and outflows - X-rays: ISM

\section{Introduction}

Herbig-Haro $(\mathrm{HH})$ objects characterize the emission regions near protostars where the accretion process is still going on, often allowing us to identify the presence of a young stellar object (YSO) not observable otherwise. The $\mathrm{HH}$ objects are observed in all wavelength bands (from radio and IR to optical and UV, and also in the X-rays; see the review of Reipurth \& Bally 2001) as a chain of knots within the supersonic protostellar jets. The knotty structure has been frequently observed in the optical band in many $\mathrm{HH}$ objects and was also discovered for the first time in X-rays in HH 154 (Favata et al. 2006; Bonito et al. 2008), one among the nearest and most luminous X-ray emitting protostellar jets. The HH 154 jet is one of the best studied, because it has been observed through multi-wavelength and multi-epoch observations with several instruments, among them the Hubble Space Telescope (HST; Fridlund \& Liseau 1998; Bonito et al. 2008), Chandra/ACIS-I (Bally et al. 2003; Favata et al. 2006), and XMM-Newton (Favata et al. 2002).

The origin of chains of optical knots commonly observed in jets have been traditionally interpreted through models of jet ejected with a sinusoidal variable velocity (e.g. Raga et al. 2007). In fact, in these models, the internal shocks resulting from plasma flowing with different velocities lead to a nodular structure, constituted by a regular chain of knots with individual proper motions. An alternative scenario has been proposed by Yirak et al. (2009) who use 3D simulations to model the appearance of clumps within the jet beam (with random locations, sizes, and speeds), and explore the possibility of intrinsic density heterogeneity of the jet. Original models of jets accounting for stochastic jets and random time variability have been previously discussed by Roberts (1986), in the context of extragalactic jets, and Raga (1992), in the limit of large distances from the stellar source. Also Vitorino et al. (2002) suggest a disk/jet launching model with a combination of a continuous and a random component of the velocity, thus justifying the random speed models.

Recently, multi-wavelength observations of HH 154 collected in 2005 with both Chandra and HST showed evidence of mutual interactions between bright knots, formed probably at different epochs and with different speeds (Bonito et al. 2008). These features also seem to be common in other objects (Caratti o Garatti et al. 2009) and are not predicted in models of jets with sinusoidal velocity. Moreover, as discussed by Yirak et al. (2009), the observed knots could be interpreted erroneously as the product of a sinusoidal variable velocity jet.

The aim of this work is to use detailed numerical modeling to investigate the origin and dynamics of the irregular knotty structure observed in several bands (and, in particular, in X-rays) 
in protostellar jets and the possible interactions between knots. Moreover, we aim at deriving general model predictions to be compared with observations of X-ray emitting protostellar jets (DG Tau, HH 2, etc.) to understand the physical mechanisms leading to X-ray emission in different objects.

In our previous studies, we investigated the origin of X-ray emission from protostellar jets, through a model of a continuously ejected supersonic jet (Bonito et al. 2004, 2007). Such a model predicts the propagation of a compact X-ray source through the circumstellar medium with spectral behavior, X-ray luminosity, and proper motion in good agreement with the observations (Favata et al. 2002). However, the model does not reproduce the complex knotty morphology and variability of the X-ray source detected in HH 154 (Favata et al. 2006).

Here we suggest an improvement to our previous model by considering the scenario of a pulsed jet interacting with an initially homogeneous ambient medium. The model presented here describes a jet constituted by a train of plasma blobs randomly ejected by the stellar source. The initial velocity of our model follows an exponential distribution, and this choice corresponds to a distribution of many low-speed blobs and a few blobs with high initial ejection velocity. The blobs are expected to interact with each other leading to the formation of knots with individual proper motions, possibly observable in various bands. In the following, we refer to blobs to consider the plasma ejected from the stellar source and to knots to consider the observable structures within the jet.

We plan to present our results in two papers. In the present paper we describe the dynamics and energetics of the pulsed jet/ambient interaction, exploring the influence of the ejection rate of plasma blobs. In the second paper (Bonito et al. 2010, in preparation) we will focus on the variability and morphology of $\mathrm{X}$-ray emitting features predicted by the model as they would be observed with Chandra/ACIS-I and with XMM/Newton.

The paper is organized as follows. In Sect. 2 we describe the numerical model of the pulsed jet, its initial setup, and the exploration of the parameter space; in Sect. 3 we analyze the hydrodynamic evolution of the model, focusing on the internal structures occurring within the jet, and the physical effects due to the thermal conduction process; in Sect. 4 we discuss the results and their physical interpretation; in Sect. 5 we draw our conclusions.

\section{The model}

The model describes a protostellar jet ramming with a supersonic variable ejection speed into an initially homogeneous ambient medium. The fluid is assumed to be fully ionized ${ }^{1}$ with a ratio of specific heats $\gamma=5 / 3$. The model takes into account the radiative cooling from optically thin plasma and the thermal conduction (including the effects of heat flux saturation). The propagation of the jet through the surrounding medium is modeled by numerically solving the time-dependent hydrodynamic equations

$$
\begin{aligned}
& \frac{\partial \rho}{\partial t}+\nabla \cdot \rho \boldsymbol{v}=0 \\
& \frac{\partial \rho \boldsymbol{v}}{\partial t}+\nabla \cdot \rho \boldsymbol{v} \boldsymbol{v}+\nabla p=0 \\
& \frac{\partial \rho E}{\partial t}+\nabla \cdot(\rho E+p) \boldsymbol{v}=-\nabla \cdot q-n_{\mathrm{e}} n_{\mathrm{H}} P(T)
\end{aligned}
$$

1 See Bonito et al. (2007). with

$$
p=(\gamma-1) \rho \epsilon, \quad E=\epsilon+\frac{1}{2}|\boldsymbol{v}|^{2},
$$

where $p$ is the pressure, $E$ the total gas specific energy (internal energy, $\epsilon$, and kinetic energy) respectively, $\rho$ is the mass density, $t$ the time, $\boldsymbol{v}$ the plasma velocity, $q$ the heat flux, $n_{\mathrm{e}}$ and $n_{\mathrm{H}}$ are the electron and hydrogen density, respectively, $P(T)$ is the radiative losses function per unit emission measure ${ }^{2}, T$ the plasma temperature.

Following Dalton \& Balbus (1993), we use an interpolation expression for the thermal conductive flux, which allows for a smooth transition between the Spitzer (Spitzer 1962) and saturated (Cowie \& McKee 1977) conduction regimes

$q=\left(\frac{1}{q_{\mathrm{spi}}}+\frac{1}{q_{\mathrm{sat}}}\right)^{-1}$,

where

$q_{\mathrm{spi}}=-\kappa(T) \nabla T$

where $\kappa(T)=9.2 \times 10^{-7} T^{5 / 2} \operatorname{erg~s}^{-1} \mathrm{~K}^{-1} \mathrm{~cm}^{-1}$ is the thermal conductivity, and

$q_{\mathrm{sat}}=-\operatorname{sign}(\nabla T) 5 \phi \rho c_{\mathrm{s}}^{3}$,

where $\phi \sim 0.3$ (Giuliani 1984; Borkowski et al. 1989, and references therein) and $c_{\mathrm{s}}$ is the isothermal sound speed.

The hydrodynamic equations are solved using the FLASH code (Fryxell et al. 2000) with customized numerical modules for the optically thin radiative losses and the thermal conduction (see Orlando et al. 2005, for the details of the implementation of the thermal conduction). The FLASH code uses a directionally split piecewise parabolic method (PPM) solver to handle compressible flows with shocks (Colella \& Woodward 1984), the PARAMESH library to handle adaptive mesh refinement (MacNeice et al. 2000), and the message-passing interface library to achieve parallelization.

\subsection{Numerical setup}

We solved the hydrodynamic equations in $2 \mathrm{D}$, using cylindrical coordinates in the plane $(r, z)$, and assuming axisymmetry with the jet axis coincident with the axis of symmetry $(z)$. The computational grid size was $(2000 \mathrm{AU} \times 6000 \mathrm{AU})$. The maximum spatial resolution achieved by our simulations is $\approx 8 \mathrm{AU}$, as determined with the algorithm of the PARAMESH library for 4 refinement levels, which corresponds to covering the initial jet radius with 4 points at the maximum resolution ${ }^{3}$.

We followed the evolution of the jet for a time interval ranging between 100 and 400 yrs, depending on the set of model parameters. The pulsed jet consists of a train of blobs, each lasting for $0.5 \mathrm{yr}$, with an ejection rate corresponding to a time interval between two consecutive blobs $\Delta t$. Each blob is ejected with a random velocity directed along the jet axis following an exponential distribution

$v(r=0, t)=v_{\mathrm{j} \operatorname{MAX}}(b-\log (\operatorname{rand}(t)-a))$,

$2 P(T)$ is described by a functional form that takes into account freefree, bound-free, bound-bound and 2 photons emission (see Raymond \& Smith 1977; Mewe et al. 1985; Kaastra \& Mewe 2000).

3 The maximum resolution corresponds to $\approx 8$ times the resolution of Chandra/ACIS-I observations and to about twice the spatial resolution of HST, $0.4^{\prime \prime} \approx 60 \mathrm{AU}$ at $150 \mathrm{pc}$ and $0.1^{\prime \prime} \approx 15 \mathrm{AU}$ at $150 \mathrm{pc}$, i.e. at the distance of the nearest star-forming region in Taurus. 
R. Bonito et al.: Generation of radiative knots in a randomly pulsed protostellar jet. I.

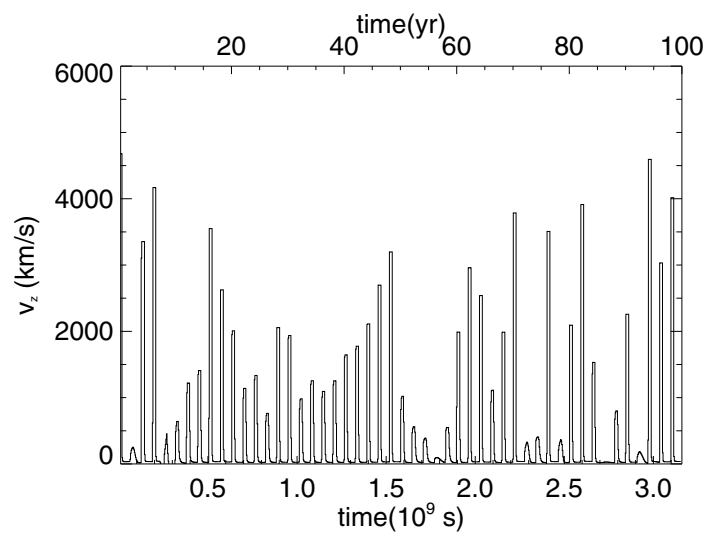

Fig. 1. Initial jet velocity of each blob as a function of time for the $\Delta t=$ 2 yr case (run LJ2).

where

$b=\log \frac{1}{1-\mathrm{e}^{-1}}$,

$a=\frac{\mathrm{e}^{-1}}{\mathrm{e}^{-1}-1}$

and rand is a number ranging between 0 and 1 obtained using a random number generator. The maximum velocity of the blobs is $v_{\mathrm{j} \text { MAX }}=4680 \mathrm{~km} \mathrm{~s}^{-1}$ and corresponds to the highly supersonic initial Mach number $M=1000$. Figure 1 shows the initial velocity values of each blob ejected with an ejection rate corresponding to $\Delta t=2 \mathrm{yr}$. We generated the random seed once for all the runs so the values follow always the same sequence, only the rate is different.

The initial random velocity of each blob is along the $z$ axis, coincident with the jet axis, and its radial profile is

$v(r, t)=\frac{v(r=0, t)}{v \cosh \left(r / r_{\mathrm{j}}\right)^{w}-(v-1)}$,

where $v(r=0, t)$ is the on-axis velocity, $v$ the ambient-to-jet density ratio, $r_{\mathrm{j}}$ the initial jet radius, and $w=4$ the steepness parameter for the shear layer that allows a smooth transition of the kinetic energy at the interface between the jet and the ambient medium. This layer avoids the growth of random perturbation at the jet border (Bodo et al. 1994). The density profile as a function of the radial direction is

$\rho(r)=\rho_{\mathrm{j}}\left(v-\frac{v-1}{\cosh \left(r / r_{\mathrm{j}}\right)^{w}}\right)$,

where $\rho_{\mathrm{j}}$ is the jet density (see also the velocity and density profiles as a function of the radial distance from the axis in Bonito et al. 2007, Fig. 1).

Reflection boundary conditions are imposed along the jet axis (consistent with the adopted symmetry), inflow boundary conditions at the base for $r<r_{\mathrm{j}}$, where $r$ is the radial distance in cylindrical coordinates and $r_{\mathrm{j}}$ is the jet radius, and outflow boundary conditions elsewhere.

\subsection{Parameter space}

The model parameters are derived from the optical and X-ray data analysis of protostellar jets (e.g. Podio et al. 2006; Bonito et al. 2008; Favata et al. 2006) and from the results of previous
Table 1. Summary of the initial physical parameters characterizing the simulations.

\begin{tabular}{lcccccc}
\hline \hline Run & $\begin{array}{c}\Delta t \\
\mathrm{yr}\end{array}$ & $v$ & $M$ & $\begin{array}{c}v_{\mathrm{j}} \\
{\left[\mathrm{km} \mathrm{s}^{-1}\right]}\end{array}$ & $\begin{array}{c}T_{\mathrm{j}} \\
{\left[10^{4} \mathrm{~K}\right]}\end{array}$ & $\begin{array}{c}n_{\mathrm{a}} \\
{\left[\mathrm{cm}^{-3}\right]}\end{array}$ \\
\hline LJ0.5 & 0.5 & 10 & 1000 & $10-4680$ & $10^{4}$ & 5000 \\
LJ2 & 2 & 10 & 1000 & $10-4680$ & $10^{4}$ & 5000 \\
LJ8 & 8 & 10 & 1000 & $10-4680$ & $10^{4}$ & 5000 \\
LJ2RD & 2 & 10 & 1000 & $10-4680$ & $10^{4}$ & 5000 \\
HJ2 & 2 & 0.1 & 300 & $10-1400$ & $10^{2}$ & 50 \\
\hline
\end{tabular}

models (Bonito et al. 2004, 2007). Table 1 summarizes the physical parameters characterizing the simulations: $\Delta t$ is the time between two consecutive blobs, $v$ is the ambient-to-jet density contrast, $M$ is the initial jet Mach number, $v_{\mathrm{j}}$ is the velocity of each ejected blob (here we indicate the range of values randomly generated in our model), $T_{\mathrm{j}}$ is the initial jet temperature, $n_{\mathrm{a}}$ is the ambient density.

The exploration of the parameter space mainly focuses on the ejection rate of the blobs composing the jet. In all our simulations, we imposed the initial radius and density of the blobs, namely $r_{\mathrm{j}} \approx 30 \mathrm{AU}$ and $n_{\mathrm{j}}=500 \mathrm{~cm}^{-3}$, respectively. For the sake of completeness, we explore both the initial light jet scenario (a jet initially less dense than the unperturbed ambient medium), and the initial heavy jet scenario (a jet initially denser than the ambient medium). We consider, therefore, initial ambient-to-jet density ratio $v=n_{\mathrm{a}} / n_{\mathrm{j}}=0.1,10$ in the heavy and light jet cases, respectively. The initial blob temperature $T_{\mathrm{j}}$ ranges between $10^{2}$ and $10^{4} \mathrm{~K}$, while the ambient temperature, $T_{\mathrm{a}}$, is derived assuming initial pressure equilibrium between the jet and the unperturbed ambient.

The initial jet Mach number values are $M=v_{\mathrm{j}} / c_{\mathrm{a}}=300$ in the heavy jet case and 1000 in the light jet case, and the latter corresponds to a maximum velocity of the first blob of about $5000 \mathrm{~km} \mathrm{~s}^{-1}$. We are interested on the evolution of the pulsed jet after a transient phase, i.e. after the first ejected blob of plasma has perturbed the ambient (which is no longer homogeneous). We therefore require a high speed of the first blob to quickly perturb the computational domain (6000 AU). The speed of each ejected blob ranges from about $10 \mathrm{~km} \mathrm{~s}^{-1}$ to $5000 \mathrm{~km} \mathrm{~s}^{-1}$ during the evolution of the pulsed jet. It is worth noting that the apparently high values of the maximum ejection velocity cannot be compared directly to the observable speeds of the knots within the protostellar jets. In fact we have chosen the initial speeds which lead, once the interaction between the ejected plasma and the ambient medium has taken place, to the observed knots speed of a few hundred $\mathrm{km} \mathrm{s}^{-1}$ (Fridlund \& Liseau 1998; Bonito et al. 2008).

Since the optical knots in HH jets, in general, show timescale variability of a few years (see morphological evolution of the $\mathrm{HH} 154$ jet in about two years discussed in Bonito et al. 2008), with new knots appearing at the base of the jet in about two years (e.g. HH 1 and HH 34, see the movies discussed in Hartigan 2003), we chose an initial ejection rate parameter corresponding to a time interval $\Delta t=2 \mathrm{yr}$ between two consecutive blobs. Then we explored this parameter by considering a $1 / 4$ value $(\Delta t=0.5 \mathrm{yr})$ and a $\times 4$ value $(\Delta t=8 \mathrm{yr})$ to constrain the ejection rate from the comparison between our model predictions and the observations. 


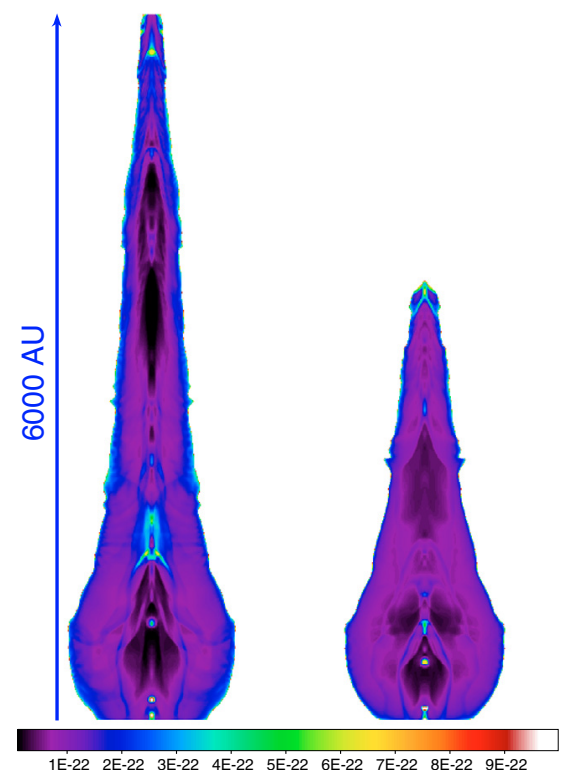

Fig. 2. 2D spatial distributions of the density of the jet/ambient interaction in runs LJ2 (left semi-panel) and LJ8 (right semi-panel).

\section{Results}

\subsection{Hydrodynamic evolution}

In all our simulations, the jet propagates through the homogeneous ambient medium, forming a cocoon, and shows a complex density structure inside. The ejection rate determines the energy powering the jet and, in fact, the maximum distance from the driving source reached at a given epoch in runs LJ0.5, LJ2, and LJ8 varies strongly. As shown in Fig. 2, the low ejection rate case (LJ8) travels through $\gtrsim 3000 \mathrm{AU}$ in $100 \mathrm{yr}$, while the high ejection rate cases reach the $6000 \mathrm{AU}$ distance in about $90 \mathrm{yr}$ (LJ2) or even less than $50 \mathrm{yr}$ (LJ0.5), because the kinetic power released in these cases is much higher than in LJ8.

Although the ambient medium is initially homogeneous, it becomes quickly inhomogeneous because the first and consecutive blobs ejected by the stellar source interact with it. Figure 3 shows a cut along the jet axis of the density (upper panel) and of the temperature (lower panel) distributions. The chain of knots within the jet due to previously ejected blobs lead to a complex structure of the medium where the new blobs are ejected. The whole computational domain (6000 AU) is filled with several structures, with the density varying by more than two orders of magnitude. Also, the thermal conditions vary along the jet axis, so that the sound speed and the Mach number are not uniform. As a result, strong variations of the shock velocities $\left(v_{\mathrm{sh}} \propto \rho^{-1 / 2}\right)$ and of the post-shock properties are determined by the varying pre-shock conditions. At variance with the model of a continuous jet, in our pulsed jet model, the density contrast between the ambient medium and the blob can vary during the jet/ambient evolution. As discussed above, in a few years, the medium is so complex that, even if the initial density of the ejected blobs does not vary $\left(n_{\mathrm{j}}=500 \mathrm{~cm}^{-3}\right.$, see the red dashed line superimposed in the upper panel of Fig. 3), the ambient density varies strongly during the evolution, thus leading to several different scenarios in which the ejected blob could be denser or less dense than the surrounding medium (see upper panel in Fig. 3).

It is worth emphasizing that the random velocity of the blobs ejected at different epochs together with the strongly inhomogeneous medium in which each blob (except the first one) propagates, lead to complex mutual interactions of the blobs. The
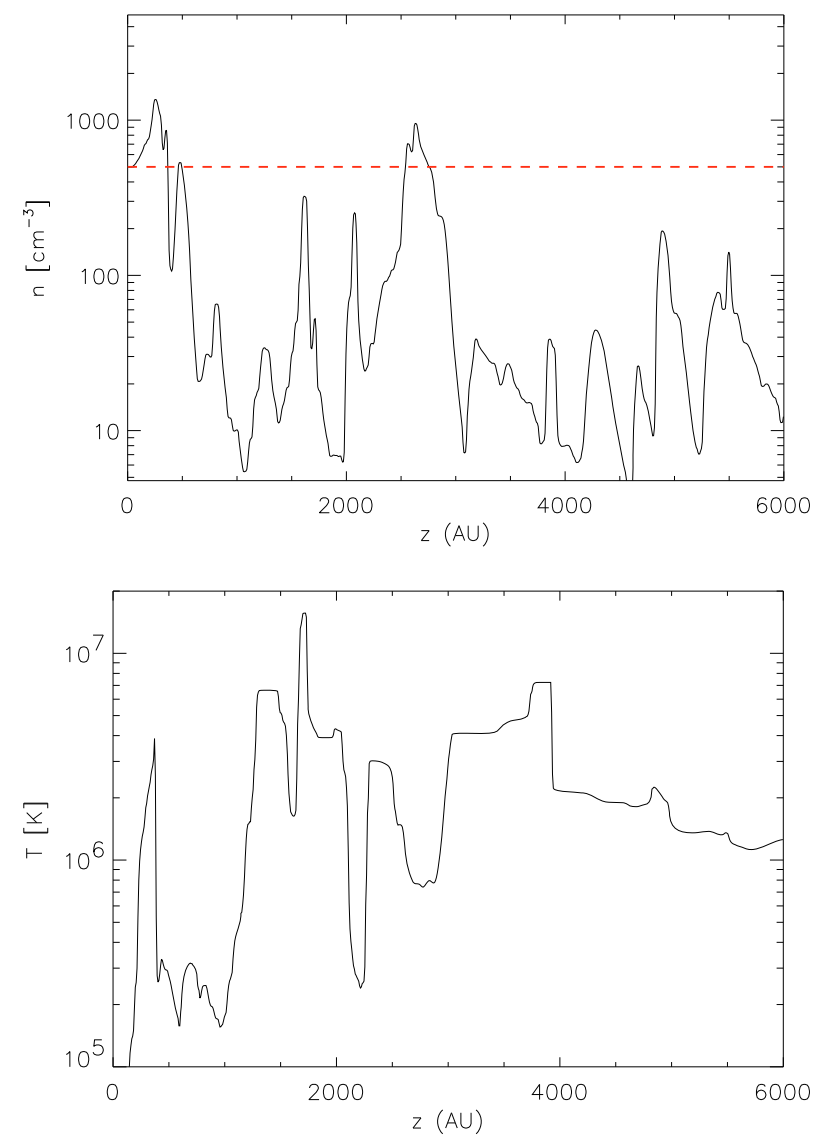

Fig. 3. Cut along the jet axis of the spatial distributions of density (upper panel) and of temperature (lower panel) in run LJ0.5. The red dashed line superimposed on the density profile indicates the initial jet density value $\left(n_{\mathrm{j}}=500 \mathrm{~cm}^{-3}\right)$.

frequency of mutual interactions within the jet depends on the ejection rate, in particular the largest is found in run LJ0.5 and the lowest in run LJ8. These interactions result in a rich variety of plasma structures that cannot be described by the current models of jet and that determine an irregular pattern of knots. In the following, we illustrate the common features that may contribute to determining the morphology of the pulsed jet by analyzing the density and temperature spatial distributions and evolution.

The most evident feature predicted by our model is an irregular chain of knots aligned along the jet axis. Depending on the model parameters, the knots may emit mainly in either the optical band or the X-ray band. In the former case, the chain of (optical) knots can be highlighted by deriving density maps of plasma with temperature ranging between (5000-100000) K, which is a proxy for optical emission. To this end, we first reconstructed the 3D spatial distributions of mass density and temperature, according to the symmetry of the problem; then, assuming the jet traveling perpendicular to the line-of-sight (LoS), we integrated the density along the LoS and derive the 2D density maps. As an example, Fig. 4 shows an enlargement of the base of the jet for run $\mathrm{HJ} 2$, i.e. in the heavy jet scenario (see Table 1), where a chain of (optical emitting) knots within the jet is visible. These knots show a detectable proper motion in a few years, in good agreement with typical observations of the moving optical knots within protostellar jets. In particular, by measuring the spatial displacement of the center of the circular region identifying the E knot in Fig. 4 in the time interval (45-50) yr, we derived an average knot speed along the jet axis of about $600 \mathrm{~km} \mathrm{~s}^{-1}$, in 
R. Bonito et al.: Generation of radiative knots in a randomly pulsed protostellar jet. I.

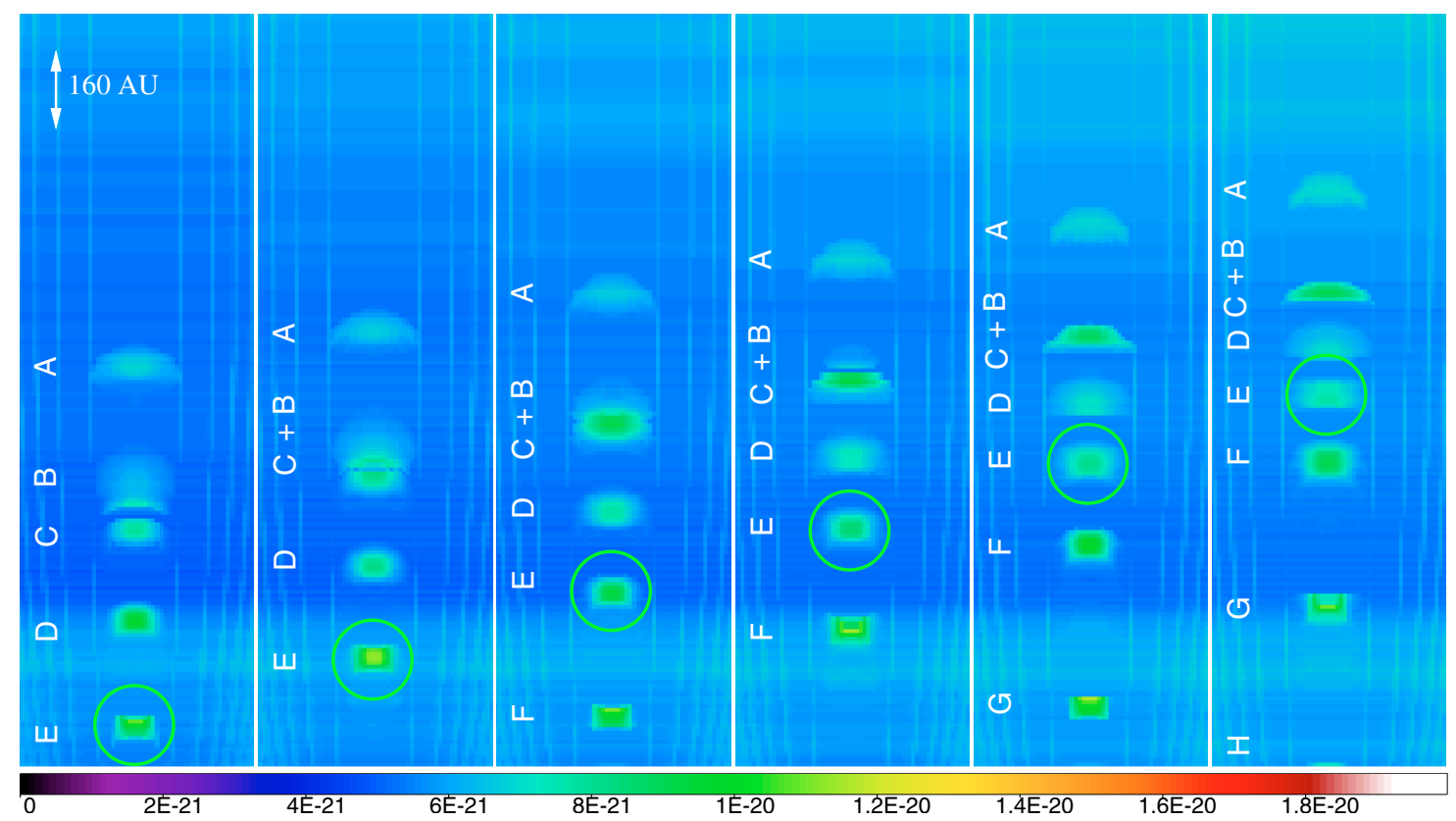

Fig. 4. Density maps of plasma with temperature ranging between (5000-100 000) K (a proxy of optical emission) for the run HJ2. The green circle (of radius $\approx 80 \mathrm{AU}$ ) superimposed on each frame marks the position of a specific knot as it evolves in time: the proper motion is detectable in a few years, the time lapse between two consecutive frames is $1 \mathrm{yr}$.

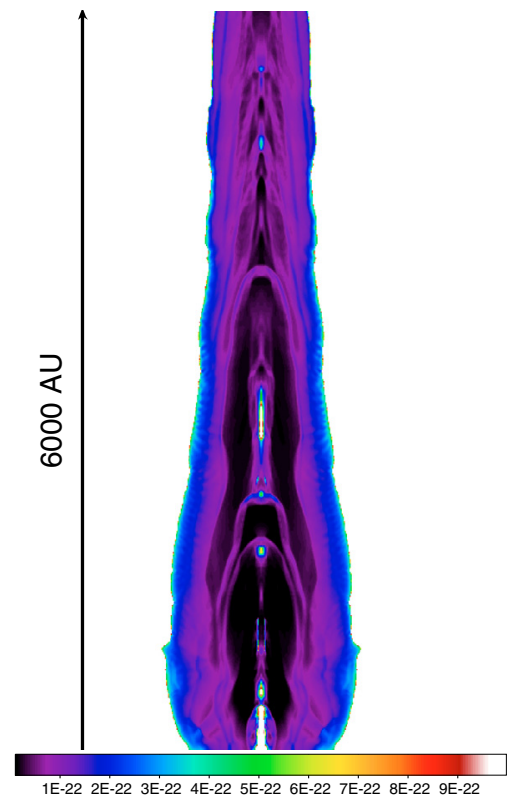

Fig. 5. 2D spatial distribution of the density of the jet/ambient interaction in run LJ0.5.

nice agreement with the values obtained from the optical observations of the knots within protostellar jets (Fridlund et al. 2005; Bonito et al. 2008; Eislöffel et al. 1994). In this case, we find evidence of knotty structures analogous to those observed in protostellar jets, with a chain of several subsequent knots in a few hundred AU from the stellar source.

Similar patterns of knots, but emitting mainly in the X-ray band (see Bonito et al. 2010, in preparation), are found in the light jet scenario when the ejection rate is high $(\Delta t=0.5,2 \mathrm{yr})$, as shown in the 2D spatial distribution of mass density in Fig. 5. In particular several dense structures are visible within the jet, mainly on the jet axis. In fact, a chain of several knots arises due to blobs ejected at different epochs.
The spatial separation between two consecutive knots is neither uniform in space nor constant in time in all the different cases analyzed because of the variation in the blob speed and in the medium surrounding the blob. This leads to a complex pattern that is consistent with observations of several protostellar jets, among them HH 154 (see Bonito et al. 2008). Sinusoidal ejection velocity models usually show a chain of knots with an almost regular pattern (Raga et al. 2007) within the jet, which does not reproduce the observed structure of complex jets, (e.g. HH 1, Reipurth et al. 2000; HH 111, Hartigan et al. 2001).

The knots resulting from the interactions among ejected blobs have, in general, different proper motions and may ultimately interact with each other. In some cases, high-speed knots can interact with the head of the jet, possibly overtaking the cocoon, hence traveling into the unperturbed ambient medium. A random variability in the injection velocity of the blobs and the consequent catching-up processes between knots have been discussed previously by Raga (1992). This effect is evident in Fig. 4, where the initially separated $C$ and $B$ knots merge in a single structure (named $C+B$ in the figure). Analogously, the density cut along the jet axis shows that, in a few years, the proper motion of the knots within the jet, the generation of new knots, and the interaction between knots are visible, and in particular indicates that the two peaks $C$ and $B$ at $t \approx 45 \mathrm{yr}$ of evolutionary stage (upper panel of Fig. 6), correspond to a single peak $C+B$ after $5 \mathrm{yr}$ (lower panel of Fig. 6). These features have been observed in several HH jets (Bonito et al. 2008; Caratti o Garatti et al. 2009).

Analogous clump-clump interactions and high-speed knots overtaking the head of the jet (including the case of off-axis structures) have been found by Yirak et al. (2009), although these authors have not considered any proxy of optical emission as done here. However the knots in Yirak et al. (2009) are the result of imposed inhomogeneities of the jet flow (describing the effect of possible instabilities), and their characteristic size is lower than the jet diameter (subradius), whereas in our model the knots are the result of the pulsed ejection of the jet (possibly 
A\&A 511, A42 (2010)
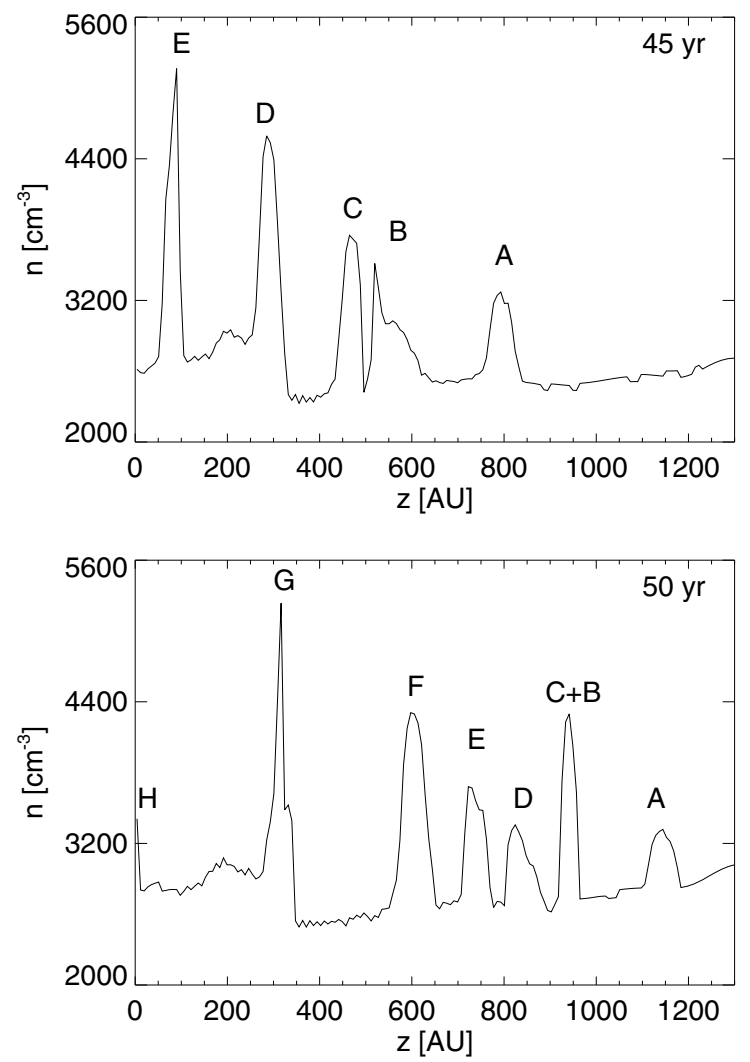

Fig. 6. Cuts along the jet axis of the first (upper panel) and the last (lower panel) frame of Fig. 4, corresponding to $45 \mathrm{yr}$ and $50 \mathrm{yr}$ since the beginning of the jet/ambient interaction.

related to accretion phenomena) and their size is comparable to the jet diameter. Note, also, that Yirak et al. (2009) considered ranges of temperatures and velocities lower than those discussed here, and their model does not take the thermal conduction effects into account. As discussed below in Sect. 3.2, our simulations show that the thermal conduction plays a crucial role in damping out the hydrodynamic instabilities that would develop within the jet, leading to a faster and more collimated jet.

Another important feature produced by our model is associated with the formation of reverse shocks moving in the opposite direction with respect to the ejected blobs and interacting with these (see Fig. 7). Under certain circumstances, this interaction produces the formation of shocks with no detectable proper motion on time-scale of a few years, as observed, for instance, in the X-ray source of HH 154 (Favata et al. 2006). The collision between knots and reverse shocks is a feature common of all the ejection rates discussed here and occurs several times during the time covered in our simulations. As an example, Fig. 7 shows one of these occurrences for run LJ0.5. In the first panel, the reverse shock is located $\approx 1200$ AU above the source driving the jet, and the knot traveling towards it is initially at about $200 \mathrm{AU}$ from the source. As the jet evolves, the knot reaches a distance of $\approx 800$ AU from the source, with the reverse shock just $\approx 200 \mathrm{AU}$ above it. After about two years, the two structures interact at a distance of $\approx 1000$ AU from the source, and the shock location does not change appreciably on a time-scale of a few years.

A more complex morphology arises when the reverse shock is reflected obliquely by the cocoon, leading, in this case, to an oblique shock pattern. Figure 8 shows the evolution of an oblique shock in a few years, moving from the external cocoon toward the jet axis, as indicated by the arrows superimposed on each frame. The propagation of the oblique shock causes the formation of a dense and well-collimated core within the jet itself as evident in the last frame of Fig. 8.

\subsection{Effects of the thermal conduction}

Our model takes into account both the radiative losses from an optically thin plasma and the thermal conduction, in Spitzer or in saturated regimes. It is interesting, therefore, to investigate the effects of the thermal conduction on the dynamics and energetics of the jet. These effects are highlighted by comparing simulations either with or without the thermal conduction. To this end, we performed an additional run with a setup identical to the one used for run LJ2 but without the thermal conduction (run LJ2RD). We find that, in run LJ2RD, the maximum distance from the stellar source is significantly shorter than in run LJ2. As an example, Fig. 9 shows the temperature distributions in the two cases after the first blob has reached the upper boundary of the computational domain, namely at $t \approx 90 \mathrm{yr}$ in LJ2 and at $t \approx 350 \mathrm{yr}$ in LJ2RD. Ignoring the effects due to the thermal conduction, the whole computational domain is fully perturbed along the jet axis after about $350 \mathrm{yr}$. We found, therefore, that the jet travels more slowly into the ambient medium if the thermal conduction effects are inhibited. In fact, in LJ2RD, the jet evolution is dominated by thermal and hydrodynamic instabilities that are known to contribute to the jet breaking (see right panel in Fig. 9). Conversely, these instabilities are damped out by the thermal conduction in run $\mathrm{LJ} 2$, and the jet propagates to greater distances.

The comparison between the two temperature maps of runs LJ2 and LJ2RD in Fig. 9 also indicate significant differences in the morphology because of the thermal conduction. In fact, besides the different evolutionary stages needed in the two cases in order to reach the top of the computational domain, it is also evident that the thermal conduction leads to a smooth and more collimated spatial distribution of temperature. The radiative case LJ2RD shows a significant radial spread of the temperature distribution, accounting for the decelerated material traveling into the instabilities.

\section{Discussion}

\subsection{Observable proper motion}

As discussed in Bonito et al. (2007), the high initial jet velocity is not an observable since we can only measure the speed of formed knots, i.e. the velocity of the plasma structures formed by the interactions among the blobs or of the blobs with the inhomogeneous surrounding medium. In other words, we can observe the effect of jet/ambient interaction, not the launching region itself. We are therefore interested in deriving the average speed of the observable knots, thereby determining the internal density structure of the jet. To this end, Fig. 10 shows the density-weighted average of the $z$-component of the plasma bulk velocity within a distance of 10 pixels ${ }^{4}$ from the jet axis for the three ejection rates analyzed (runs LJ0.5, L2, L8). The velocity values are consistent with typical knots speeds observed within optical jets (few hundreds $\mathrm{km} \mathrm{s}^{-1}$, Eislöffel \& Mundt 1998; Bally et al. 2003; Bonito et al. 2008). In particular, we find very good agreement for the $\Delta t=2 \mathrm{yr}$ case, and the speed value decreases with increasing $\Delta t$ parameter.

Following our model results, we can make predictions about the initial ejection velocity leading to the observed speed. To

\footnotetext{
${ }^{4}$ Corresponding to a physical radius of $\approx 80 \mathrm{AU}$.
} 
R. Bonito et al.: Generation of radiative knots in a randomly pulsed protostellar jet. I.

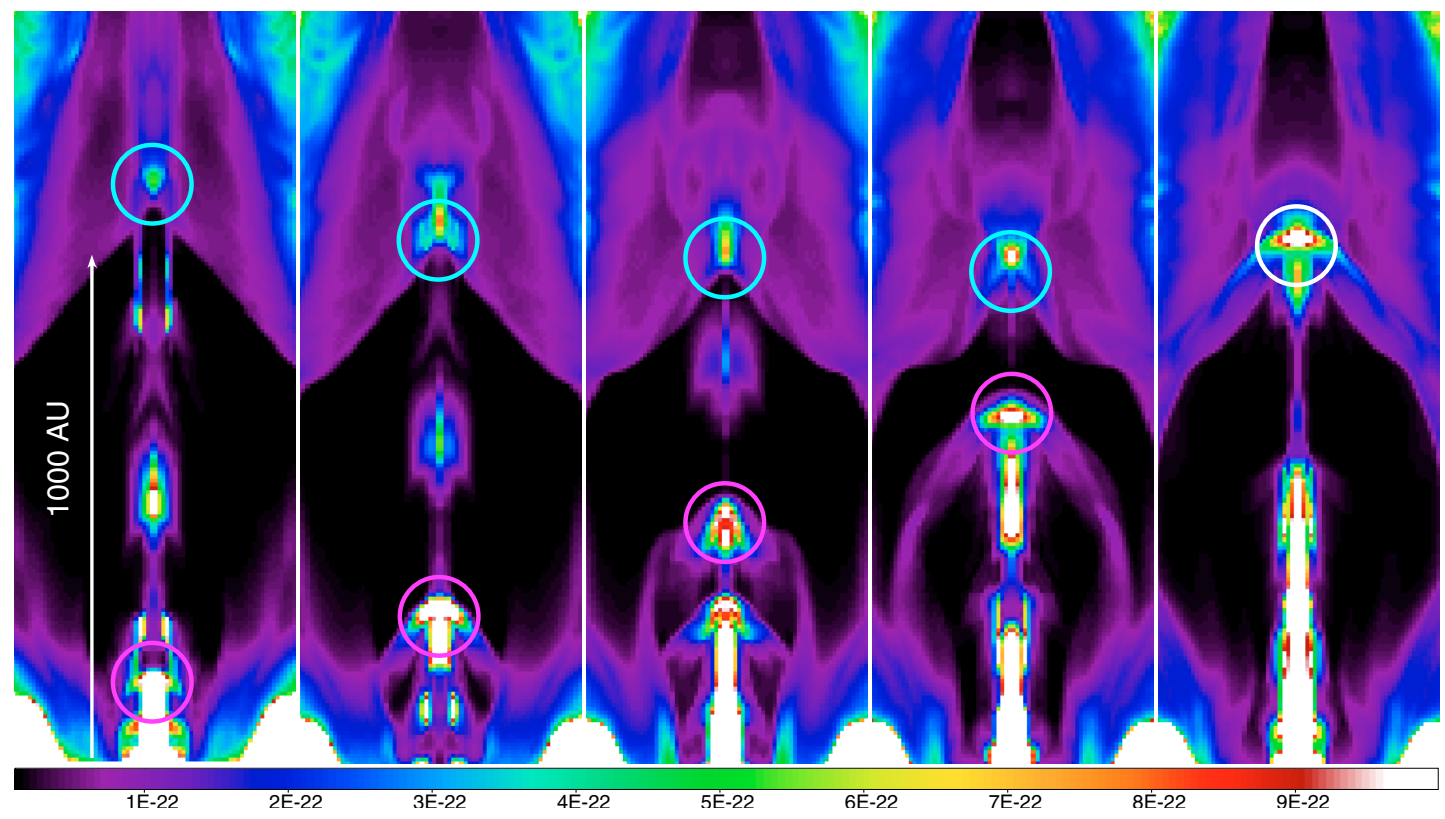

Fig. 7. Evolution in a few years of the 2D spatial distributions of the density of the jet/ambient interaction in run LJ0.5: reverse shocks (highlighted by the cyan circle in each panel) form and travel in the direction opposite the ejected blobs (highlighted by the magenta circle in each panel) and interact with them (white circle superimposed on the last panel).

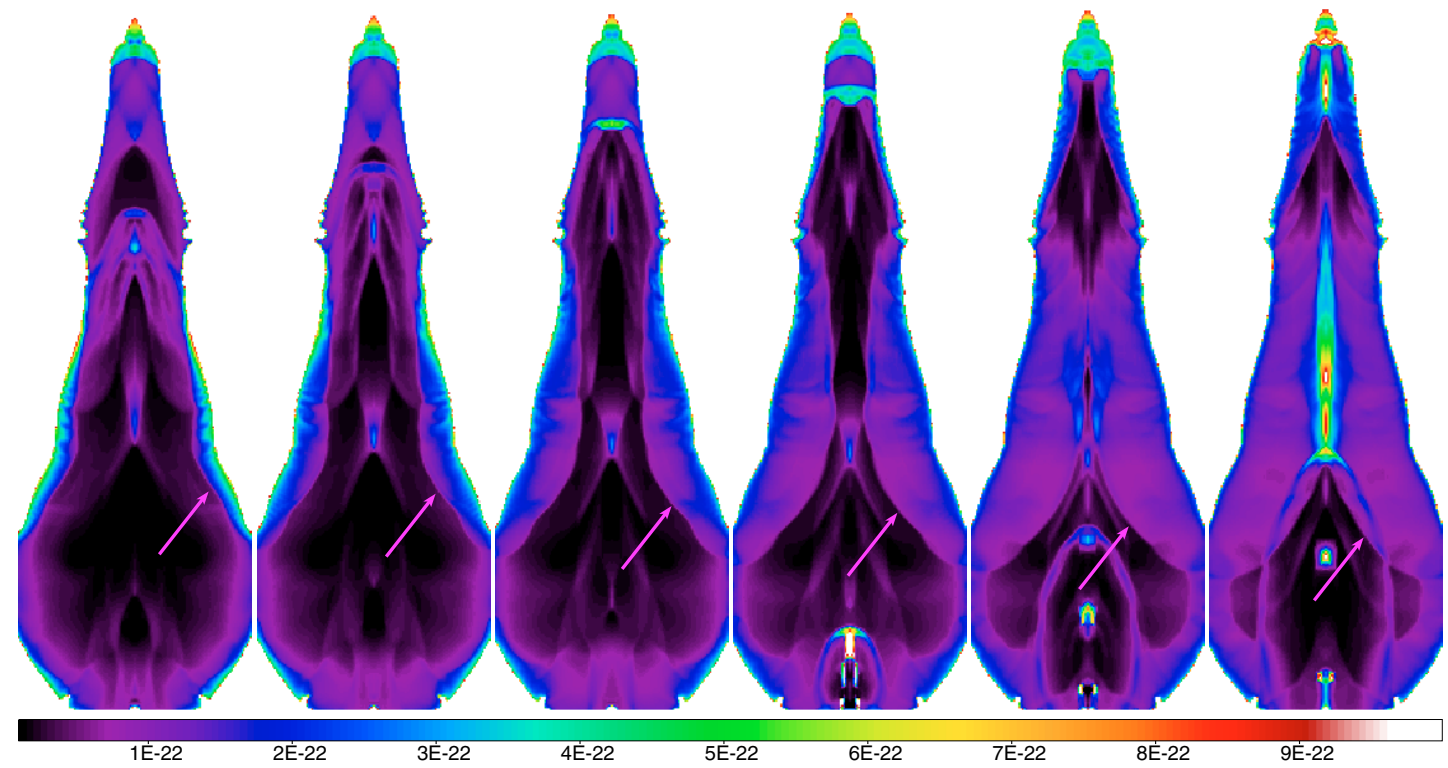

Fig. 8. Run LJ8: evolution of the 2D spatial distributions of the density of the jet/ambient interaction (the first frame is after $70 \mathrm{yr}$ and the last after $75 \mathrm{yr}$ since the beginning of the jet/ambient interaction). Oblique shocks form (marked by the arrows in each panel) as the ejected blob interacts with the lateral cocoon.

verify that our assumed values are reasonable, we can compare the mechanical luminosity derived from our simulations with the observed bolometric luminosity of the source of the HH 154 jet. In particular, from the values of the initial velocity used here and shown in Table 1 and by following relation (18) in Bonito et al. (2007), the kinetic power derived from our model ranges between $L_{\text {mech }} \sim 10^{-7}-10 L_{\odot}$, i.e. always below the observed bolometric luminosity of the source of the HH 154 jet, $L_{\mathrm{bol}} \approx$ $40 L_{\odot}$ (see Bonito et al. 2007, Table 1). Thus we conclude that the initial blob velocity values assumed in our models lead to reasonably low kinetic power.

Finally, following the relation (17) from Bonito et al. (2007), we can derive a jet mass-loss rate ranging between $\dot{M} \sim 10^{-11}-10^{-9} M_{\odot} /$ yr. Once again, as discussed in detail in
Bonito et al. (2007), these results concerning both the kinetic power and the mass-loss rate are several orders of magnitude lower than those observed in $\mathrm{CO}$ outflows, so it seems reasonable that the simulated protostellar jets cannot drive molecular outflow.

\subsection{Ejection rate of the blobs}

Our simulations show that observable knots are mainly produced by the mutual interactions among blobs of plasma. We therefore expect that the frequency of interactions between knots depends on the ejection rate of the blobs (see Sect. 2). In fact, from our exploration of the parameter $\Delta t$, we find that the knot interactions occur quite frequently in runs with high ejection rates (LJ05, 
A\&A 511, A42 (2010)

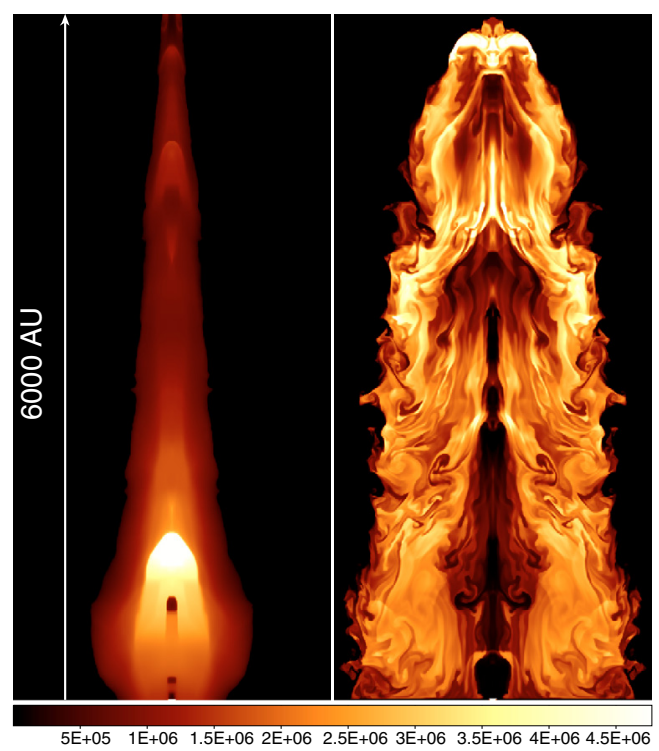

Fig. 9. 2D spatial distributions of the temperature of the jet/ambient interaction in the $\Delta t=2 \mathrm{yr}$ case: with radiative losses and thermal conduction after about $90 \mathrm{yr}$ (left panel) and the pure radiative case after about $350 \mathrm{yr}$ (right panel) of evolution.

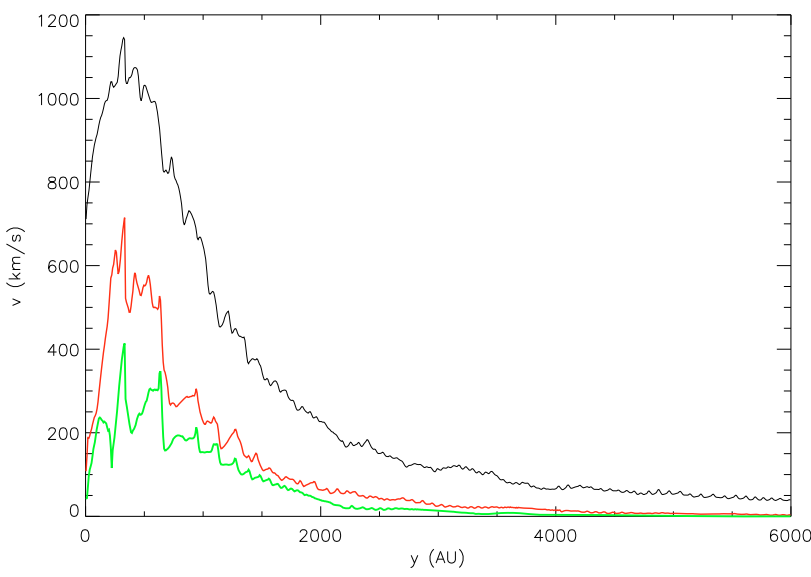

Fig. 10. Average speed along the jet axis for the three runs LJ0.5, LJ2, and LJ8: the $\Delta t=0.5 \mathrm{yr}$ case is in black, the $\Delta t=2 \mathrm{yr}$ case is in red, and the $\Delta t=8 \mathrm{yr}$ case is in green.

and $\mathrm{LJ} 2$ ), whereas it is almost absent in the low ejection rate case (LJ8) on the time-scale analyzed here. This result can be understood from the following simple estimation.

Bonito et al. (2007) showed that the observable velocity of a knot is a fraction of the initial jet velocity. This result is confirmed by our simulations. As discussed in Sect. 4.1, Fig. 10 shows that the maximum average speed of the knots along the jet axis is a fraction of the initial average blob velocity $\left(\approx 2000 \mathrm{~km} \mathrm{~s}^{-1}\right)$ and the values of the knots speed derived from the runs are consistent with optical observations. The time at which we expect to observe a collision between two consecutive knots is

$t_{\mathrm{coll}}=\frac{v_{\mathrm{knot}}}{\Delta v_{\mathrm{knot}}}(\Delta t)_{\mathrm{knot}}$

where $\Delta v_{\mathrm{knot}}$ and $(\Delta t)_{\mathrm{knot}}$ are the average relative speed and the time interval between two consecutive knots, respectively. Both $v_{\text {knot }}$ and $\Delta v_{\text {knot }}$ are calculated assuming the same deceleration factor (since each knot velocity is a fraction of the initial blob speed). The latter is evaluated by considering only the blobs ejected with higher speed with respect to the previous ones. Since knots are produced mainly by the mutual interaction between blobs and between blobs and the ambient, we expect that $(\Delta t)_{\mathrm{knot}} \gtrsim \Delta t$. Considering $\Delta t$ instead of $(\Delta t)_{\mathrm{knot}}$ in Eq. (12), we have, therefore, a lower limit on the collision time between knots. We find that the interactions rises on average over about $t_{\text {coll }}=10,30$, and more than $100 \mathrm{yr}$ in runs LJ0.5, LJ2, and LJ8, respectively. This estimate explains the presence of knot interactions in the high ejection rate cases (LJ0.5 and LJ2) and the absence in the low ejection rate case (LJ8) on the time-scale considered. In the light of the above discussion, we suggest, therefore, that the ejection rate might be constrained if the frequency of mutual interactions among ejected blobs can be determined from observations. The constrain of the ejection rate could be also interesting in the context of the accretion scenario. In fact, it is believed that the ejection/accretion phenomena are intimately related, but a full description of this relation is still lacking.

\section{Conclusions}

We investigated the physical mechanism determining the irregular knotty structure of protostellar jets commonly observed in the optical band, and recently also detected in X-rays (e.g. HH 154, Favata et al. 2006). To this end, we developed a numerical model describing a pulsed jet, consisting of a chain of blobs, each ejected along the jet axis with random initial velocity, and traveling in an initial homogeneous ambient medium. Our simulations represent the first attempt to model a pulsed jet that simultaneously includes the thermal conduction and the radiative losses processes. In particular, we find that the thermal conduction plays a crucial role in damping out the hydrodynamic instabilities that would develop within the cocoon. Since these instabilities contribute to breaking the jet, the thermal conduction makes the jet propagate to greater distances.

The jet is modeled both as an initial light jet (a scenario that seems likely to describe the observations of $\mathrm{HH} 154$, both in X-rays and in the optical band; Bonito et al. 2008) and as an initial heavy jet. In all the cases, we find that the interactions among subsequent ejected blobs are a common feature of our simulations. As a result of these interactions, each new ejected blob travels through a strongly inhomogeneous medium characterized by density and thermal conditions largely varying along the jet axis; as a result, the density contrast between the blob and the surrounding medium varies during the jet/ambient evolution and the different pre-shock conditions leads to strong variations of the shock velocities and of the post-shock properties.

The mutual interactions among the blobs lead to a variety of density structures within the jet not described by current models of jets (however see Yirak et al. 2009). These structures determine a rather complex morphology of the jet. The most relevant are (i) single high-speed knots, showing a measurable proper motion that nicely agrees with optical and X-rays observations; (ii) irregular chains of knots aligned along the jet axis, and possibly interacting with each other, consistent with observations of several protostellar jets; (iii) reverse shocks interacting with outgoing knots; (iv) oblique shock patterns produced by the reflection of shocks at the cocoon surrounding the jet. We also found that the range of velocity of the knots formed from mutual interactions among blobs is consistent with the typical range of values observed (e.g. Reipurth \& Bally 2001).

We explored the effects of the ejection rate of the blobs on the evolution of the density and temperature spatial distributions of the jet/ambient interaction. The frequency of interactions between formed knots depends on the ejection rate. We suggest, 
therefore, that the comparison between our model results and the observations may give some hints to the ejection rate of protostellar jets. Since the opposite and complementary processes of accretion of mass onto a star and ejection of mass from a star are commonly assumed to be related, we may also infer important physical information on the accretion rates from the constraints on the ejection rate.

We expect some details of our model to depend on the assumption of the exponential distribution for the ejection velocity of each blob (determined with a given random seed). From a quantitative point of view, the single blob velocity can change with a different random seed and also the energetics and dynamics of particular structures are expected to vary if different velocity distributions are chosen. Nevertheless, we expect to obtain the main features discussed here, namely the formation of shock fronts, the collision among knots due to blobs ejected at different epochs with different speed, the interaction among knots, and reverse shocks.

The model of a randomly ejected pulsed jet is, therefore, a promising scenario for explaining the physical mechanism leading to the irregular knotty structure observed within protostellar jets in a wide range of wavelengths from optical to X-rays. Further development of this work will be reported in a forthcoming paper (Bonito et al. 2010, in preparation) where we will investigate the morphology and variability of X-ray emitting structures formed within the randomly ejected pulsed jet.

Acknowledgements. We would like to thank the referee, Dr. A. C. Raga, for his helpful comments and suggestions. R.B., S.O., M.M, and G.P. acknowledge support by the Marie Curie Fellowship Contract No. MTKD-CT-2005029768. The software used in this work was developed in part by the DOEsupported ASC/Alliances Center for Astrophysical Thermonuclear Flashes at the University of Chicago, using modules for thermal conduction and optically thin radiation constructed at the Osservatorio Astronomico di Palermo. The calculations were performed at CINECA (Bologna, Italy) and on the cluster at the SCAN (Sistema di Calcolo per l'Astrofisica Numerica) facility of the INAF - Osservatorio Astronomico di Palermo. This work was supported in part by Agenzia Spaziale Italiana under contract No. ASI-INAF I/088/06/0.

\section{References}

Bally, J., Feigelson, E., \& Reipurth, B. 2003, ApJ, 584, 843

Bodo, G., Massaglia, S., Ferrari, A., \& Trussoni, E. 1994, A\&A, 283, 655

Bonito, R., Orlando, S., Peres, G., Favata, F., \& Rosner, R. 2004, A\&A, 424, L1

Bonito, R., Orlando, S., Peres, G., Favata, F., \& Rosner, R. 2007, A\&A, 462, 645

Bonito, R., Fridlund, C. V. M., Favata, F., et al. 2008, A\&A, 484, 389

Borkowski, K. J., Shull, J. M., \& McKee, C. F. 1989, ApJ, 336, 979

Caratti o Garatti, A., Eislöffel, J., Froebrich, D., et al. 2009, A\&A, 502, 579

Colella, P., \& Woodward, P. R. 1984, Journal of Computational Physics, 54, 174

Cowie, L. L., \& McKee, C. F. 1977, ApJ, 211, 135

Dalton, W. W., \& Balbus, S. A. 1993, ApJ, 404, 625

Eislöffel, J., \& Mundt, R. 1998, AJ, 115, 1554

Eislöffel, J., Mundt, R., \& Böhm, K. 1994, AJ, 108, 1042

Favata, F., Fridlund, C. V. M., Micela, G., Sciortino, S., \& Kaas, A. A. 2002, A\&A, 386, 204

Favata, F., Bonito, R., Micela, G., et al. 2006, A\&A, 450, L17

Fridlund, C. V. M., \& Liseau, R. 1998, ApJ, 499, L75

Fridlund, C. V. M., Liseau, R., Djupvik, A. A., et al. 2005, A\&A, 436, 983

Fryxell, B., Olson, K., Ricker, P., et al. 2000, ApJS, 131, 273

Giuliani, J. L. 1984, ApJ, 277, 605

Hartigan, P., Morse, J. A., Reipurth, B., Heathcote, S., \& Bally, J. 2001, ApJ, 559, L157

Hartigan, P. M. 2003, in Rev. Mex. Astron. Astrofis. Conf. Ser. 15, ed. J. Arthur, \& W. J. Henney, 112

Kaastra, J. S., \& Mewe, R. 2000, in Atomic Data Needs for X-ray Astronomy, 161

MacNeice, P., Olson, K. M., Mobarry, C., de Fainchtein, R., \& Packer, C. 2000, Computer Physics Communications, 126, 330

Mewe, R., Gronenschild, E. H. B. M., \& van den Oord, G. H. J. 1985, A\&AS, 62,197

Orlando, S., Peres, G., Reale, F., et al. 2005, A\&A, 444, 505

Podio, L., Bacciotti, F., Nisini, B., et al. 2006, A\&A, 456, 189

Raga, A. C. 1992, MNRAS, 258, 301

Raga, A. C., de Colle, F., Kajdič, P., Esquivel, A., \& Cantó, J. 2007, A\&A, 465, 879

Raymond, J. C., \& Smith, B. W. 1977, ApJS, 35, 419

Reipurth, B., \& Bally, J. 2001, ARA\&A, 39, 403

Reipurth, B., Heathcote, S., Yu, K. C., Bally, J., \& Rodríguez, L. F. 2000, ApJ, 534,317

Roberts, D. A. 1986, ApJ, 300, 568

Spitzer, L. 1962, Physics of Fully Ionized Gases (New York: Interscience)

Vitorino, B. F., Jatenco-Pereira, V., \& Opher, R. 2002, A\&A, 384, 329

Yirak, K., Frank, A., Cunningham, A. J., \& Mitran, S. 2009, ApJ, 695, 999 